Communications in Physics, Vol. 25, No. 1 (2015), pp. 51-57

DOI:10.15625/0868-3166/25/1/5669

\title{
MONTE CARLO SIMULATION ON THE PHASE TRANSITION OF ANIMAL GROUP
}

\author{
NGUYEN PHUOC THE \\ Department of Natural Science, Duy Tan University, \\ K7/25 Quang Trung, Hai Chau, Da Nang, Vietnam \\ NGO VAN THANH \\ Institute of Physics, Vietnam Academy of Science and Technology, \\ 10 Dao Tan, Ngoc Khanh, Ba Dinh, Hanoi, Vietnam
}

Received 08 December 2014

Accepted for publication 28 March 2015

\begin{abstract}
In this work, we proposed a new model in order to study the phase transition behaviour of animal group. The individuals in the group are considered as the XY spins which can move freely in a plane with the same speed. For the interactions between every pairs of the individuals, we used the Hamiltonian of the system in the model of spin current with including a long-range Morse potential. Using the Monte-Carlo simulation technique, we obtained the main parameters of the model: the averaged velocity of all individuals which is equivalent to the order parameter or magnetization for the spin model, and the concentration of the individuals. The simulation result clearly shows that the system has three phases which correspond to the uncollected, flocking (schooling) and runaway behaviors at very low, medium and high noise, respectively. The simulation predictions are in good agreement with experimental observations [B. L. Partridge, Sci. Am. 246, (1982) 114].
\end{abstract}

Keywords: flocking behaviors, phase transition, Monte-Carlo simulation.

\section{INTRODUCTION}

One of the most familiar and intriguing examples of many degrees of freedom, non-equilibrium dynamical system is a flocking or schooling behavior, which has been a phenomenon of long standing interest. Well-known examples are found in populations such as large schools of fish, the gatherings of birds, the swarming of ants and the herding of sheep [1-5]. The schooling or flocking is a behavior of some animal species where they stay together in a group for social reasons. They derive many benefits from these behaviors including defence against predators, easier to move, enhanced foraging success and higher success in finding a mate.

The group of animal does not need a leader or an external stimulus to avoid splitting up, move cohesively and adopt a common direction. Therefore, these behaviors can be described by a self-organized system. A mathematical model is proposed by Cucker and Smale [6] (CSM) using the equations of classical mechanics. In which, the interaction is introduced in the form of attraction or repulsion force which depends on the distance between each individual and its neighbors. However, the equation of motion in CSM is a discrete function of distance [7-9]. On 
the other hand, T. Vicsek et. al. introduced a physical model [10] which has been extensively developed during the last 20 years [11-13]. This model is a novel type of dynamics in order to investigate phase transition in non-equilibrium systems based on the ferromagnetic spin model. In which, the velocity and direction of the particles is determined by a simple rule: at each time step, a given particle moves with a constant velocity, a new direction is obtained by the average direction of all the neighborhood particles within a circle of radius $R$ with including a random noise. The effects of vision angle on the phase transition has been investigated [14], the simulation result showed that the schooling behavior is only for the prey species, but not for the predator species. However, all above models are applicable to study the phase transition at high noise only, where the system is changed from the ordered phase to the disordered phase.

In this paper, we propose a simple model of self-organized system which is combined of Vicsek's model [10] and the model of spin current [15]. The Hamiltonian of this system is written in terms of exchange interaction, chemical potential and long-range Morse potential [16]. The phase transition behavior is investigated by using the Monte-Carlo (MC) technique, we obtained the dependence of the order parameter and the concentration of the individuals on the external noise. These quantities could be used to explain the behaviors of animal group such as uncollected behavior at very low noise, flocking or schooling behavior at medium noise and runaway behavior at high noise.

The paper is organized as follows, Section II is devoted to the description of the model and the algorithm of MC simulation. Section III shows the phase transition behavior obtained from the simulations. Concluding remarks are given in Sec. IV.

\section{THE MODEL AND TECHNIQUES}

We consider a shelf-organized system consists of $N$ individuals continuously move in a plane $(x, y)$ of linear size $L$ with the maximum velocity $v_{0}$. The $i$ th individual is characterized by their position $\boldsymbol{r}_{i}=\left(x_{i}, y_{i}\right)$ and orientation $\boldsymbol{S}_{i}$ of magnitude $S=1$. The orientation or the moving direction of each individual is the same as a classical XY spin with ferromagnetic interaction. The Hamiltonian of the system is defined by

$$
\mathscr{H}_{\text {tot }}=\mathscr{H}_{e}+\mathscr{H}_{M}+\mathscr{H}_{c}
$$

with

$$
\begin{aligned}
\mathscr{H}_{e} & =-\sum_{<i, j>} J_{i j}\left(\boldsymbol{S}_{i} \cdot \boldsymbol{S}_{j}\right), \\
\mathscr{H}_{M} & =K_{0} \sum_{<i, j>}\left[e^{-2 a\left(r_{i j}-r_{0}\right)}-2 e^{-a\left(r_{i j}-r_{0}\right)}\right], \\
\mathscr{H}_{c} & =D_{0} \sum_{i}\left(n_{i}-n_{0}\right) .
\end{aligned}
$$

In which, $\mathscr{H}_{e}$ is the exchange energy of the system, $S_{i}$ is the orientation of $i$ th individual at position $\boldsymbol{r}_{i}$. The interaction factor $J_{i j}$ is given by

$$
J_{i j}=J_{0} e^{-r_{i j}}
$$

with $J_{0}>0$ and $r_{i j}$ is the distance between two individuals $S_{i}$ and $S_{j} . \mathscr{H}_{M}$ is a long-range interaction which has the form of Morse potential with $K_{0}, a$ and $r_{0}$ being the constants. The chemical 
potential $\mathscr{H}_{c}$ depends on the concentration of the individuals. $n_{i}$ is the number of individuals in a circle of radius $r_{c}^{D}$, centered at $i$ th individual. $n_{0}=\pi\left(r_{c}^{D}\right)^{2} N / L^{2}$ is the average of individuals in an unit of area.

In Eqs. (2) and (3), $\sum_{<i, j>}$ indicates the sum over every individual pairs $\boldsymbol{S}_{i}$ and $\boldsymbol{S}_{j}$ inside a circle centered at $S_{i}$. We denote $r_{c}^{e}$ and $r_{c}^{M}$ being the cut-off radii of exchange interaction and Morse potential, respectively.

Let us explain the biological meaning of the terms in the Hamiltonian (1). $\mathscr{H}_{e}$ is the interaction for the alignment of the individuals, they have the same orientation in the flocking state. $\mathscr{H}_{M}$ is an attractive potential for long range of distance between the individuals, it helps to the animals moving close to each other. The last term $\mathscr{H}_{c}$ is a strong repulse interaction, so the individuals are not be curdled.

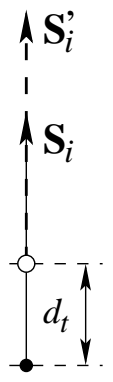

(a)

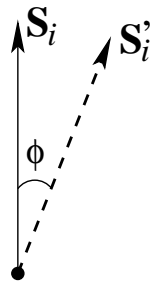

(b)

Fig. 1. The forward movement (a) and the rotation (b) of an individual.

In order to investigate the nature of the phase transition, we use the standard Monte-Carlo method with Metropolis algorithm for updating both position and orientation of the individuals. Here we define a noise parameter $\xi$ which is equivalent to the temperature in statistical physics. The way the simulation algorithm is implemented can be described by a scheme:

(1) Choose an initial state

(2) Choose an individual $i$ at position $r_{i}$ with orientation $S_{i}$

(3) Calculate the energy $E_{i}$

(4) Try to changes the position by a forward movement in a random length $d_{t}$ generated from the uniform distribution on an interval $\left[0, v_{0}\right]$. Its new position $\boldsymbol{r}_{i}$ (see in Fig. 1a) is obtained by

$$
\begin{aligned}
x_{i}^{\prime} & =d_{t} \cos \theta_{i}, \\
y_{i}^{\prime} & =d_{t} \sin \theta_{i}
\end{aligned}
$$

with the orientation angle

$$
\theta_{i}=\arctan \left(y_{i} / x_{i}\right)
$$

(5) Calculate the new energy $E_{f}$

(6) Generate a random number $R$ such that $0<R<1$

(7) If $R<\exp \left[-\left(E_{f}-E_{i}\right) / \xi\right]$, accept the translational motion and update the energy $E_{i} \rightarrow$ $E_{f}$ 
(8) Generate a random angle $\phi$ from a zero-centered Gaussian distribution on the interval $[-\pi, \pi]$. Try to changes the orientation $S_{i}^{\prime}($ Fig. 1b) as follows

$$
\begin{aligned}
& \left(S_{i}^{x}\right)^{\prime}=\cos \theta_{i}^{\prime}, \\
& \left(S_{i}^{y}\right)^{\prime}=\sin \theta_{i}^{\prime},
\end{aligned}
$$

where

$$
\theta_{i}^{\prime}=\theta_{i}+\phi
$$

(9) Calculate the new energy $E_{f}$

(10) Generate other random number $R$

(11) If $R<\exp \left[-\left(E_{f}-E_{i}\right) / \xi\right]$, update the orientation

(12) Go to the next individual and go to (3)

In MC simulations, we calculate the averaged order parameter $Q$ and the concentration of the individuals which are defined as

$$
\begin{aligned}
Q & =\frac{1}{N}\left\langle\left|\sum_{i=1}^{N} S_{i}\right|\right\rangle, \\
\rho & =\frac{1}{N}\left\langle\sum_{i=1}^{N} n_{i}\right\rangle .
\end{aligned}
$$

\section{SIMULATION RESULTS}

In biology, all the individuals of an animal group are spread to find foods if there is no danger. In this situation, the individuals are distributed in the space and out of alignment with a small concentration. For simplicity, we say the group of animals is in "uncollected" behavior. When the animals are faced with danger such as predators, their natural instinct is to flee not fight, they use their natural herding instinct to bind together in a small area for safety with the same orientation and maximum concentration. This stage is called the "flocking" behaviour. All individuals of group will move further from predator in the same direction and then stampede as fast as they can when being under predator's attack. At the final stage, they are in the "runaway" behavior. Any external excitations endanger animal lives are so-called the "noise". In summary, almost of animal groups have three behaviors:

- Uncollected behavior at low noise, where the individuals are distributed in the space with disordered orientation.

- Flocking behavior at medium noise, they are bind together with the ordered orientation and high concentration.

- Runaway behavior at high noise, all individuals stampede in the different directions. They are distributed in the space with the disordered orientation.

In the ferromagnetic spin systems, the ordering of the system is quantified by the order parameter $Q$ which is defined in Eq. (10). When all spins are parallel, the order parameter reaches to the maximum $Q=1$, so we say the system is in the ordered phase. Whereas, the system is in the disordered phase, where the spins have different values, therefore the order parameter is about zero. On the other hand, the quantity $\rho$ in the Eq. (11) characterizes to the concentration of the 
spins. Depending on the noise, the spins are close to each other or distributed in the space, hence the concentration $\rho$ is high or low.

Now we apply the algorithm of MC technique to the new model which have been described in Sec. II. For the model's parameters, we use here the exchange interaction constant $J_{0}=1.0$ and cut-off radius $r_{c}^{e}=1.0$. For the Morse potential: $K_{0}=0.2, a=0.5, r_{0}=3.0$ and $r_{c}^{M}=3.0$. The parameters of chemical potential are $D_{0}=0.05$ and $r_{c}^{D}=1.0$. The maximum velocity is set to be a constant $v_{0}=0.05$.

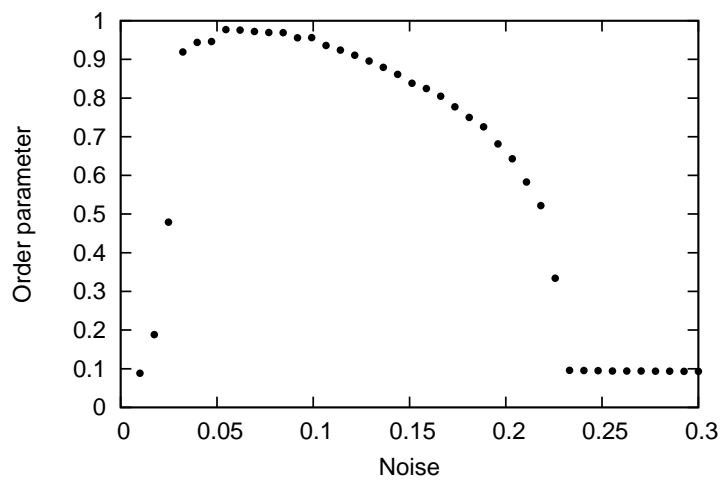

Fig. 2. Order parameter versus noise $\xi$ with the system size $N=100$.

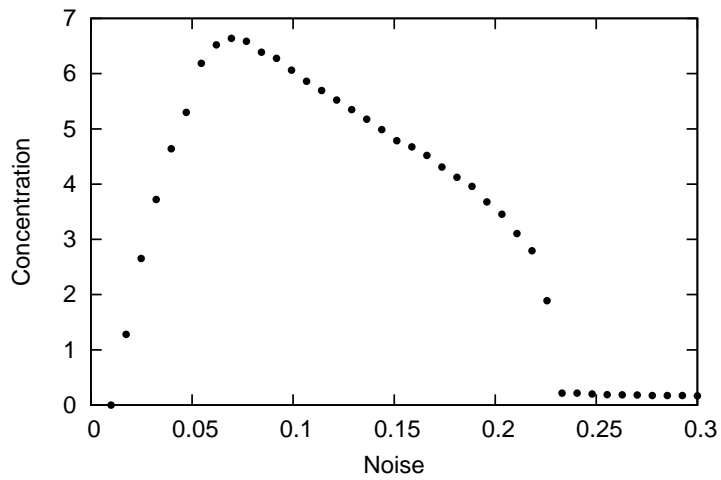

Fig. 3. The concentration of the individuals versus noise $\xi$.

For the simulations, we generate an initial state with $N$ individuals are randomly distributed on a square of size $L \times L$, where $N=100$ and $L=40$. The orientation angle $\theta_{i}$ of each individual is randomly chosen in an interval $[0 \div 2 \pi]$. At each $\xi$, the equilibration time lies around $4 \times 10^{6} \mathrm{MC}$ steps per individual and we compute statistical averages over $8 \times 10^{6} \mathrm{MC}$ steps per individual. Periodic boundary conditions (PBCs) are used in the $x y$ plane. We obtained the dependence of the order parameter and the concentration on the noise which are shown in the figures 2 and 3 , respectively.

We see that, the system undergos two transition points at low noise $\xi_{c}^{1}=0.025$ and high noise $\xi_{c}^{2}=0.225$. The transition points separate the system into three phases: phase I, II and III at low noise $\xi<0.025$, medium noise $0.025 \leq \xi \leq 0.225$ and high noise $\xi>0.225$, respectively. 
In phase $\mathrm{I}$, the order parameter $Q$ is about 0.2 and $\rho \simeq 1.0$, the system is in the disordered phase with the distributed position of the individuals. Fig. 4 clearly shows that the individuals have been distributed in the space with different orientation. Therefore, this phase is equivalent to the uncollected behavior of animal group.

With increasing the noise, the order parameter $Q$ increases up to 1.0 and the concentration $\rho$ increases to 6.64 . The system undergos from disordered phase (phase I) to ordered phase (phase II) over the first critical point $\xi_{c}^{1}=0.025$, then one slowly decreases in phase II until the noise reaches to the second critical point $\xi_{c}^{2}=0.225$. In this phase, the individuals are close to each other with the same orientation (see in Fig. 5). The ordered phase corresponds to the flocking behavior of animal group when they are faced with danger such as predators.

In the case of high noise, the system is changed from ordered phase (phase II) to disordered phase (phase III) passing through the second critical point. With increasing the noise, the order parameter quickly decreases to 0.1 and the concentration decreases to zero. Fig. 6 shows the snapshot of the system at high noise $\xi=0.233$ where the individuals are located at the positions far away from each other with random orientations. The phase III is equivalent to the runaway behavior of animal group when they are under predator's attack.

Let us say a few words on the phase transition, the discontinuity in order parameter curve is a clear signature of a first-order transition. Of course, it is only one of the conditions for the first-order transition. To give a conclusion on that, ones have to calculate the critical exponent quantities and the energy histogram with a double peak, but it is not our purpose here.

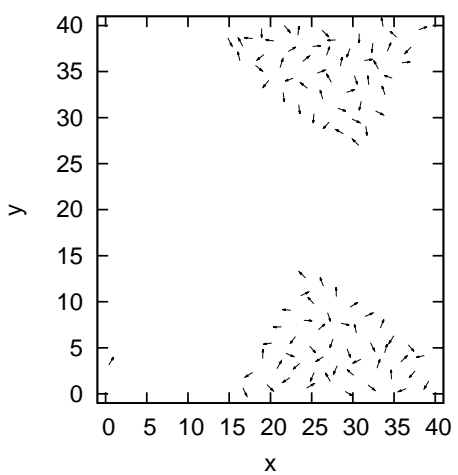

Fig. 4. Snapshot of the system at very low noise $\xi=0.01$, the arrows indicate the position and orientation of each individual.

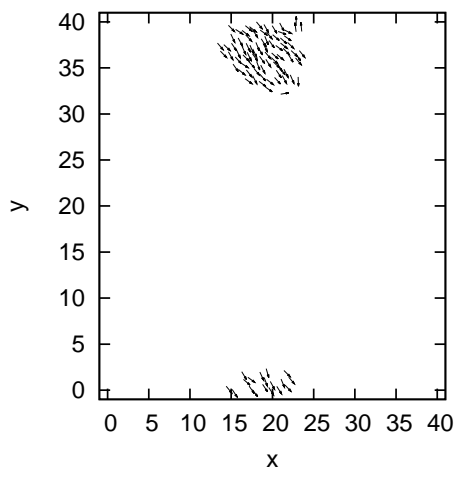

Fig. 5. Snapshot of the system in the flocked state at $\xi=0.039$.

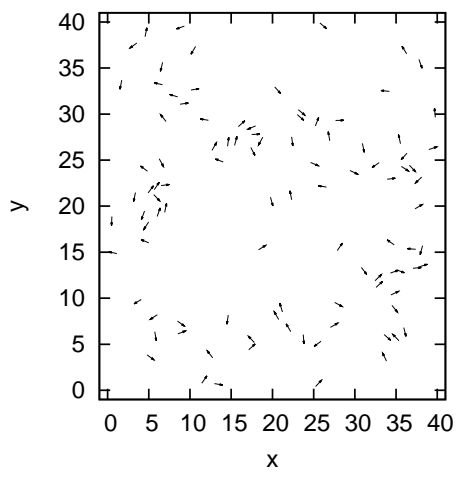

Fig. 6. Snapshot of the system at high noise $\xi=0.233$. 


\section{CONCLUDING REMARKS}

We have introduced in this paper the simple model for studying the phase transition behavior of animal group. The group of animals is considered as a system of self-propelling particles. Using the definitions for the velocity of the individuals in Vicsek's model combining with the model of spin current, we established the Hamiltonian of the system with including the Morse potential for long range interaction. We also presented in detail the algorithm for the Monte-Carlo simulation technique.

The result presented here will serve as a testing for the new model. We showed that the system has three phases and it goes through two phase transitions at very low noise and high noise. These phases correspond to the behaviors of animal group: uncollected, flocking and runaway. Our results are in good agreement with the experiment observations [17], where the behavior of animal group changes from the uncollected behavior to flocking behavior at low noise and then changes to the runaway behavior at high noise. The main goal of our model is applicable to investigate the transition from disordered phase to ordered phase at low noise. Note that all previous models are only proposed for the study on the phase transition at high noise, i.e., from ordered phase to disordered phase. For developing the model, we will study the system size dependence of the phase transition. It is the purpose of the future works.

\section{ACKNOWLEDGMENTS}

This work was supported by the Nafosted (Vietnam National Foundation for Science and Technology Development), Grant No. 103.02-2011.55.

\section{REFERENCES}

[1] Ch. Beccoa, N. Vandewallea, J. Delcourtb, and P. Poncin, Physica A 367, (2006) 487.

[2] A. Huth and C. Wissel, Ecol. Modell., 75, (1994) 135.

[3] M. Maldonado-Coelho and M. A. Marini, Biol. Conserv. 116, (2004) 19.

[4] J. H. Furbay, Science 76, (1932) 367.

[5] W. D. Hamilton, J. Theor. Biol., 31, (1971) 295.

[6] F. Cucker and S. Smale, IEEE Trans. Automat. Control, 52, (2007) 852862.

[7] S.-Y. Ha and E. Tadmor, Kinetic and Related models, 1, (2008) 415-435.

[8] S-Y. Ha and J.-G. Liu, Commun. Math. Sci, 7, (2009) 297-325.

[9] A. Okubo, Diffusion and Ecological Problems: Mathematical Models, (Lecture Notes in Biomathematics, Vol. 10. Springer-Verlag, New York, 1980).

[10] T. Vicsek, A. Czirok, E. Ben-Jacob, I. Cohen, O. Shochet, Phys. Rev. Lett. 75, (1995) 1226.

[11] A. Czirok, H.E. Stanley, and T. Vicsek, Phys. Phys. A, 30, (1996) 137; A. Czirók, M. Vicsek, T. Vicsek, Physica A 264 (1999) 299304; A. Czirók and T. Vicsek, Physica A 281, (2000) 17;

[12] I. D. Couzin, J. Krause, R. James, G. D. Ruxton, N. R. Franks, J. Theor. Biol. 218, (2002) 1-11.

[13] T. Vicsek and A. Zafeiris, Physics Reports 517, (2012) 71.

[14] P. The Nguyen, S-. H. Lee and V. Thanh Ngo, Commu. Phys. 23, (2013) 121.

[15] K. Akabli and H. T. Diep, J. Appl. Phys. 103, (2008) 07 F307.

[16] P. M. Morse, Phys. Rev. 34, (1929) 57.

[17] B. L. Partridge, Sci. Am. 246, (1982) 114. 